\title{
Urinary triclosan concentrations and early outcomes of in vitro fertilization-embryo transfer
}

\author{
Rui Hua ${ }^{1, *}$, Yao Zhou ${ }^{1, *}$, Biao Wu ${ }^{1}$, Zhongwei Huang ${ }^{2}$, Yongtong Zhu, Yali Song ${ }^{1}$, Yanhong Yu , \\ Hong $\mathrm{Li}^{1}$ and Song Quan ${ }^{1}$ \\ ${ }^{1}$ Department of Obstetrics and Gynaecology, Nanfang Hospital, Southern Medical University, Guangzhou, \\ Guangdong, China and ' Department of Obstetrics and Gynaecology, Yong Loo Lin School of Medicine, \\ National University of Singapore, Singapore \\ Correspondence should be addressed to S Quan or H Li; Email: quansong@smu.edu.cn or lihong6918@126.com
}

$*(\mathrm{R}$ Hua and $\mathrm{Y}$ Zhou contributed equally to this work)

\begin{abstract}
Triclosan (TCS) exists ubiquitously in the environment. Several in vitro and in vivo studies have demonstrated that TCS exerts endocrine disruptive effects on reproduction, but data from human populations are limited and conflicting. The objective of our study was to investigate whether high urinary TCS concentration is adversely associated with early reproductive outcomes in women undergoing in vitro fertilization-embryo transfer (IVF-ET). This prospective cohort study was conducted from September 2015 to June 2016, including 156 infertile women undergoing their first IVF-ET cycle. Two spot urine samples were collected prior to oocyte retrieval for TCS detection using solid-phase extraction (SPE) and liquid chromatography coupled with tandem mass spectrometry (LC-MS/MS). Linear regression model and binary logistic regression model were used to evaluate the association between urinary TCS concentrations and IVF outcomes. The intake of aquaculture food may have positive influences on urinary TCS concentrations. After adjustment for age, body mass index (BMI), baseline follicle-stimulating hormone (FSH), antral follicle count (AFC) and smoking status, a significant decrease of top quality embryo formation and implantation rate was observed in patients with urinary TCS concentration greater than or equal to the median level $(0.045 \mu \mathrm{mol} / \mathrm{mol} \mathrm{Cr})$. We concluded that TCS exposure may exert negative effects during early stages of human reproduction.

Reproduction (2017) 153 319-325
\end{abstract}

\section{Introduction}

Triclosan (TCS) is a broad-spectrum antimicrobial agent being widely used in pharmaceuticals and personal care products with concentration around $0.1-0.3 \%$ (w/w) (Dhillon et al. 2015). Previously, TCS had not been considered as a chemical pollutant, leading to widespread use without proper regulation. Recently, because of its ubiquitous existence in the environment and human population (Calafat et al. 2008, Wang \& Tian 2015), especially with a molecular structure similar to anthropogenic estrogens (Dhillon et al. 2015, Wang \& Tian 2015), increasing attention has been diverted to potential toxic effects of this chemical. In humans, ingestion is the major route of TCS absorption (Moss et al. 2000, Sandborgh-Englund et al. 2006), and various reports have noted its occurrence in plasma, breast milk and urine (Allmyr et al. 2006a,b, Li et al. 2013). Approximately $80 \%$ of the orally administered dose in volunteers is excreted in the urine during the first 4 days (Sandborgh-Englund et al. 2006), and the elimination half-life of TCS is estimated to be approximately 10-20 h (Sandborgh-Englund et al. 2006, Queckenberg et al. 2010). Even though, there has been evidence demonstrating its bioaccumulation after daily use (Mustafa et al. 2003).

In vitro studies using various cell lines indicated both estrogenic (Gee et al. 2008, Huang et al. 2014) and androgenic (Ahn et al. 2008, Christen et al. 2010) activities of TCS. The reproductive endocrine-disrupting effects of TCS might be mediated either through sex hormone-related pathways or during steroidogenesis (Kumar et al. 2008, Forgacs et al. 2012). Besides, the synergistic effect of TCS with BPA (Crawford \& Decatanzaro 2012) and ethinylestradiol (Stoker et al. 2010) indicates its disruptive effects in sex hormone metabolism.

The estrogenic effect of TCS was also detected in female rats. Being exposed to TCS at the highest dose (i.e. $150 \mathrm{mg} / \mathrm{kg} /$ day) for 21 days, a significantly advanced age of vaginal opening and increased uterine weight could be observed (Stoker et al. 2010). However, several studies conducted to assess the potential androgenic 
effects of TCS produced discordant results. In a study by Zorilla and colleagues, decreased serum testosterone level was observed in male Wistar rats when exposed to a $200 \mathrm{mg} / \mathrm{kg}$ dose of TCS (Zorrilla et al. 2009), while Axelstad failed to find any androgenic effects (Axelstad et al. 2013).

The evidences of TCS's potential effects in human reproduction are still limited and conflicting. A recent Navigation Guide systematic review indicated that TCS is 'possibly toxic' to reproductive and developmental health (Johnson et al. 2016). However, several epidemiological studies indicated a weak association between TCS exposure and the age of first menarche in girls (Buttke et al. 2012), or idiopathic male infertility (Chen et al. 2013). In our study, we evaluated the association between urinary TCS concentration and early reproductive outcomes in women undergoing in vitro fertilization-embryo transfer (IVF-ET), including oocyte maturation rates, fertilization rates, top quality embryo formation and embryo implantation rates. This is one of the first studies assessing TCS endocrinedisrupting effects in IVF-ET, which is the best model to study the potential effects during the early stage of human reproduction.

\section{Materials and methods}

\section{Study participants and data collection}

In this prospective cohort study, 156 infertile patients undergoing their first IVF/ICSI cycle in the Center for Reproductive Medicine of Nanfang Hospital, Southern Medical University, were recruited from September 2015 to June 2016. Women aged 20-40 years using their own oocytes for IVF were eligible for recruitment. Cryothaw and donor egg cycles were not included in this analysis. The study was approved by the Institutional Review Board for Nanfang Hospital (study number NFEC-2015-106), and all the participants signed written informed consents.

Upon recruitment, a brief questionnaire was assigned to each patient to collect baseline data including demographics, occupation, medical history, lifestyle and residential factors. In the questionnaire, a passive smoker is defined as a person exposed to smoking at work or at home at least 1 day per week for over $15 \mathrm{~min}$. A snack is a portion of food smaller than a regular meal, generally eaten between meals, which comes in a variety of forms including packaged snack foods and other processed foods. Aquaculture food refers to both freshwater and saltwater populations, such as fish, crustaceans and mollusks. The frequency of snack intake, cosmetic use, smoked or grilled food intake and aquaculture food intake was classified as always, sometimes and never. 'Always' refers to $\geq 1-3$ times per week, 'sometimes' refers to $\leq 1-3$ times monthly and 'never' refers to no such use in the recent 3 months. Clinical information was obtained from electronic medical records.

\section{Treatment protocol and clinical IVF measures}

Patients were treated with a standard long GnRH-agonist protocol. Briefly, either long- or short-acting GnRH-agonist Reproduction (2017) 153 319-325 was injected in the mid-luteal phase. After pituitary downregulation, the ovarian stimulation was conducted till at least two follicles had reached a diameter of $\geq 18 \mathrm{~mm}$, followed by human chorionic gonadotropin (HCG) administration to induce final follicular maturation. Oocyte retrieval was performed via trans-vaginal ultrasound-guided follicle aspiration $36 \mathrm{~h}$ later. Oocytes were cultured individually from the time of retrieval until the assessment on day 3 pre-transfer. The oocyte maturation rate was defined as the total number of mature oocytes (MII oocytes for ICSI cycle and fertilized oocytes for IVF cycle) divided by the total number of oocytes retrieved. The fertilization rate was defined as the total number of fertilized oocytes divided by the total number of oocytes retrieved. The normal fertilization rate was defined as the total number of normally fertilized oocytes (2PN) divided by the total number of oocytes retrieved. A top quality embryo was characterized by the presence of 4-5 blastomeres on day 2 and $7-8$ blastomeres on day 3 , the absence of multinucleated blastomeres and $<10 \%$ cellular fragments. The top quality embryo formation rate was defined as the total number of top quality embryos divided by the total number of normally fertilized oocytes (2PN).

A maximum of three embryos were transferred on day 3 after retrieval and luteal phase support was given by daily injections of progesterone started from the day of oocyte retrieval continuing until the day of pregnancy test. A positive pregnancy test was defined by a plasma $\beta$-HCG concentration $>10 \mathrm{IU} / \mathrm{L}$. A clinical pregnancy was defined as an intrauterine gestational sac with a heartbeat 30 days after embryo transfer, and clinical pregnancy rate is defined as the total number of pregnancies divided by the total number of initial cycles. Implantation rate is defined as the total number of gestational sacs divided by the total number of embryos transferred.

\section{Blood samples and hormone assays}

Blood samples were taken on day 2-5 of the patients' spontaneous menstrual cycle before any treatment for basic measurements of follicle-stimulating hormone $(\mathrm{FSH})$, luteinizing hormone (LH), estradiol (E2) and progesterone (P4), and serum peak E2 level was measured on the day of HCG administration. Serum hormonal levels were measured by electrochemiluminescence (ECL) immunoassay (Roche).

\section{Urine sample collection and urinary TCS concentrations}

Two spot urine samples were collected from each patient, with the first one collected between day 6 of the ovarian stimulation and the day of HCG administration; the second one collected on the day of oocyte retrieval. Both samples were $15 \mathrm{~mL}$ fasting samples, and were collected in a sterile clean polypropylene bottle. Each urine sample was divided into aliquots and creatinine was measured immediately. The rest of the aliquots were stored at $-20^{\circ} \mathrm{C}$ and analyzed.

The urinary concentrations of total (free and conjugated) TCS were measured using solid-phase extraction (SPE) and liquid chromatography coupled with tandem mass spectrometry (LC-MS/MS) as described before (Provencher et al. 2014). Briefly, $4 \mathrm{~mL}$ urine was treated with $\beta$-glucuronidase/sulfatase (Sigma), and TCS was extracted on the Caliper Rapid Trace SPE 
workstation (Hopkinton, MA, USA). The analyses by LC-MS/ MS were carried out with Agilent 1100 series HPLC system. An Eclipse Plus-C18 reversed-phase column $(250 \mathrm{~mm} \times 4.6 \mathrm{~mm}$ i.d., $5 \mu \mathrm{m}$; Agilent) was applied for LC separation of analytes. The limit of detection (LOD) was $0.005 \mu \mathrm{g} / \mathrm{L}$ and the limit of quantification was $0.015 \mu \mathrm{g} / \mathrm{L}$. The recovery rate of the target substances from urine samples was detected to be higher than 95\%. TCS concentrations were adjusted by creatinine $(\mathrm{Cr})$ concentrations $(\mu \mathrm{mol} / \mathrm{L})$. The arithmetic mean of the Cr-adjusted TCS concentrations ( $\mu \mathrm{mol} / \mathrm{mol} \mathrm{Cr}$ ) from two spot urine samples was calculated and used as the TCS concentration of the patient. TCS concentrations below the LOD were assigned a value equal to the LOD divided by the square root of 2 before $\mathrm{Cr}$ adjustment.

\section{Statistical methods}

Demographic and baseline reproductive characteristics of the female patients are presented using mean \pm S.D. or percentages, and TCS concentrations of different groups are presented using median (Q1-Q3). Patients' exposures to TCS were divided into two groups by the median of urinary TCS concentration, with the one below the median considered as the reference group. Demographic and baseline reproductive characteristics between the two groups of urinary TCS concentrations were compared using $t$-test for continuous variables and chi-squared tests for categorical variables. The influences of patients' BMI and lifestyles on urinary TCS concentrations were analyzed using non-parametric test. Group comparisons were conducted using contingency table analysis. Linear regression model and binary logistic regression model were used to evaluate the association between urinary TCS concentrations and IVF outcomes. Final models were adjusted for age (continuous), BMI (continuous), baseline FSH (continuous), AFC (continuous) and smoking status (non-smoker vs passive smoker). The variables considered as potential confounders included factors previously related to IVF outcomes in this and other studies. All tests were twotailed and the level of statistical significance was set at 0.05 . Computation was performed using SPSS statistical software version 12.0.1 for Windows.

\section{Results}

\section{Demographics}

A total of 156 patients were enrolled in the study, and 12 were excluded due to failure of oocyte retrieval $(n=4)$ or only one urine sample collected $(n=8)$. The average age of the participants was $31.4 \pm 4.4$ years and $61.1 \%$ of them were diagnosed to be tubal factor infertility. The average $\mathrm{BMI}$ was $21.6 \pm 2.8 \mathrm{~kg} / \mathrm{m}^{2}$, while $17(11.8 \%)$ patients were overweight (BMI $\left.>25 \mathrm{~kg} / \mathrm{m}^{2}\right)$. Almost 123 patients went through cleavage stage embryo transfer (ET), while fresh cycle ET was cancelled in 21 patients to prevent ovarian hyperstimulation syndrome. Patients' characteristics are presented in Table 1.

According to the lifestyle questionnaire responses, none of the participants were current or ever-smokers,
Table 1 Baseline characteristics of the enrolled patients.

\begin{tabular}{lc}
\hline Characteristics & $\boldsymbol{n}(\%)$ \\
\hline Age $($ years $)$ mean \pm S.D. (range) & $31.4 \pm 4.4(21-39)$ \\
$\mathrm{BMI}\left(\mathrm{kg} / \mathrm{m}^{2}\right)$ mean \pm S.D. (range) & $21.6 \pm 2.8(16.8-34.0)$ \\
$\mathrm{BMI}>25 \mathrm{~kg} / \mathrm{m}^{2}$ & $17(11.8 \%)$ \\
Diagnosis & $102(70.8 \%)$ \\
Female factor & $5(3.5 \%)$ \\
Ovulation disorders & $9(6.3 \%)$ \\
Endometriosis & $88(61.1 \%)$ \\
Tubal factor & $37(25.7 \%)$ \\
Male infertility & $5(3.5 \%)$ \\
Unexplained & \\
Oocyte treatment & $105(72.9 \%)$ \\
IVF & $37(25.7 \%)$ \\
ICSI & $2(1.4 \%)$ \\
IVF/ICSI &
\end{tabular}

while 31 (21.5\%) were passive smokers. Almost 12.5\% (18/144) and 20.8\% (30/144) of the participants always took overcooked food (smoked or grilled food) and snacks in the recent 3 months. Almost 21.5\% (31/144) and $46.5 \%(67 / 144)$ of them were frequent aquaculture food consumers and cosmetic users respectively (Table 2). The intake of aquaculture food $(P<0.001)$ may have positive influences on urinary TCS concentrations as shown in Table 2, while the place of residence or the other lifestyle habits may not be related to TCS exposure (all $P>0.05$ ).

\section{Triclosan detection}

The detection rate of TCS was $60.4 \%$ (87/144) with the concentration ranging from below the LOD to $4.98 \mu \mathrm{mol} / \mathrm{mol} \mathrm{Cr}$. The median (Q1-Q3) and geometric mean was $0.045(0.004-0.337)$ and $0.041 \mu \mathrm{mol} / \mathrm{mol}$

Table 2 Patients lifestyle and urinary TCS concentrations $(\mu \mathrm{mol} / \mathrm{mol} \mathrm{Cr})$.

\begin{tabular}{|c|c|c|c|c|c|}
\hline Characteristics & $n$ & Median & Mean (S.D.) & Statistics & $P$ \\
\hline \multicolumn{4}{|c|}{ Place of residence } & 2.389 & 0.303 \\
\hline Downtown & 82 & 0.071 & $0.412(0.816)$ & & \\
\hline Countryside & 35 & 0.044 & $0.226(0.466)$ & & \\
\hline Suburb & 27 & 0.010 & $0.365(0.727)$ & & \\
\hline \multicolumn{4}{|l|}{ Smoking } & 1.320 & 0.187 \\
\hline Non-smokers & 31 & 0.051 & $0.530(0.780)$ & & \\
\hline $\begin{array}{l}\text { Passive } \\
\text { smokers }\end{array}$ & 113 & 0.044 & $0.311(0.710)$ & & \\
\hline \multicolumn{4}{|c|}{ Smoked or grilled food intake } & 0.489 & 0.625 \\
\hline Always & 18 & 0.050 & $0.317(0.465)$ & & \\
\hline Sometimes & 126 & 0.045 & $0.364(0.760)$ & & \\
\hline \multirow{2}{*}{\multicolumn{4}{|c|}{ Aquaculture food intake }} & & \\
\hline & & & & 15.561 & 0.000 \\
\hline Always & 31 & 0.236 & $0.709(0.941)$ & & \\
\hline Sometimes & 106 & 0.038 & $0.279(0.647)$ & & \\
\hline Never & 7 & 0.003 & $0.004(0.003)$ & & \\
\hline \multicolumn{4}{|l|}{ Cosmetic use } & -1.848 & 0.065 \\
\hline Always & 67 & 0.099 & $0.444(0.825)$ & & \\
\hline Sometimes & 77 & 0.022 & $0.283(0.629)$ & & \\
\hline Never & 0 & & & & \\
\hline \multicolumn{4}{|l|}{ Snacks intake } & -0.428 & 0.669 \\
\hline Always & 30 & 0.089 & $0.297(0.519)$ & & \\
\hline Sometimes & 114 & 0.034 & $0.374(0.775)$ & & \\
\hline Never & 0 & & & & \\
\hline
\end{tabular}


Table 3 Distribution of unadjusted and urinary creatinine-adjusted triclosan (TCS) levels.

\begin{tabular}{lccccc}
\hline & $\begin{array}{c}\text { Geometric } \\
\text { mean }\end{array}$ & $\begin{array}{c}\text { Arithmetic } \\
\text { mean } \pm \text { s.D. }\end{array}$ & $\begin{array}{c}\text { 25th } \\
\text { centile }\end{array}$ & $\begin{array}{c}\text { 50th } \\
\text { centile }\end{array}$ & $\begin{array}{c}\text { 75th } \\
\text { centile }\end{array}$ \\
\hline TCS $(\mu \mathrm{g} / \mathrm{L})$ & 0.058 & $0.628 \pm 1.159$ & 0.004 & 0.064 & 0.645 \\
$\begin{array}{c}\text { TCS/urinary } \\
(\mu \mathrm{mol} / \mathrm{mol} \mathrm{Cr})\end{array}$ & 0.041 & $0.358 \pm 0.728$ & 0.004 & 0.045 & 0.337 \\
\hline
\end{tabular}

$\mathrm{Cr}$ respectively (Table 3). Patients who had TCS concentrations at or above the median of the sample were significantly younger than those with TCS below the median (30.6 vs 32.2, $P=0.025$ ), and the former group of patients' total antral follicle count (AFC) was significantly higher (15.4 vs 11.9, $P=0.009)$. After adjustment for age, $\mathrm{BMI}$ and smoking status using linear regression model, the difference of AFC between the two groups of patients is still statistically significant $(P=0.029)$. There were no significant differences between the two groups of patients in body mass index $(\mathrm{BMI})$, menstrual cycle duration and baseline hormone levels (Table 4).

\section{Triclosan concentration and IVF outcomes}

TCS concentration and the relevant IVF outcomes are presented in Table 5 . We observed a significant increase of peak E2 level in patients with TCS concentrations at or above the median $(4129.6 \pm 2554.4$ vs $3237.9 \pm 1871.0 \mathrm{pg} / \mathrm{mL}, P=0.046$ ). As there was a moderate correlation between peak E2 and number of oocyte retrieved (Spearman $r=0.70, P<0.000$ ), without surprising, a slight increased trend was observed in the number of oocyte retrieved in patients with TCS concentrations at or above the median $(14.4 \pm 6.6$ vs
Table 5 Adverse effects of TCS exposure on early reproductive results after adjustment for age, $\mathrm{BMI}$, baseline $\mathrm{FSH}, \mathrm{AFC}$ and smoking status using Binary Logistic Regression Model.

\begin{tabular}{lccc}
\hline Outcomes & OR & $\mathbf{9 5} \% \mathbf{C I}$ & $\boldsymbol{P}$ \\
\hline Fertilization & 1.238 & $0.990-1.548$ & 0.061 \\
Top embryo formation & 1.322 & $1.046-1.698$ & 0.020 \\
Implantation & 1.709 & $1.024-2.854$ & 0.040 \\
\hline
\end{tabular}

$\mathrm{Cl}$, confidence interval; $\mathrm{OR}$, odds ratio.

$12.3 \pm 7.5, P=0.074)$. However, when adjusted for age, baseline $\mathrm{FSH}, \mathrm{BMI}, \mathrm{AFC}$ and smoking status using linear regression model, no statistical differences could be observed either in peak E2 $(P=0.298)$ or in the number of oocyte retrieved $(P=0.709)$.

Although there was no statistical difference in oocyte maturation rate $(78.8 \%$ vs $79.3 \%, P>0.05)$, a significant decrease of fertilization rate $(73.0 \%$ vs $79.9 \%, P=0.001)$, top quality embryo formation rate $(40.9 \%$ vs $48.9 \%, P=0.006)$ and implantation rate $(27.6 \%$ vs $50.0 \%, P<0.001)$ could be observed in patients with higher urinary TCS concentration (Table 4). There were no statistical differences in normal fertilization rate and clinical pregnancy rate (per cycle) between the two groups of patients. After adjustment for age, BMI, baseline FSH, AFC and smoking status using binary logistic regression model, no dose-response correlation was noted between TCS concentrations and the outcomes of fertilization $(\mathrm{OR}=1.238, \quad P=0.061)$, whereas negative doseresponse associations could be observed between TCS concentrations and top embryo formation $(\mathrm{OR}=1.332$, $P=0.020)$, as well as implantation $(\mathrm{OR}=1.709$, $P=0.040)$ (Table 5).

Table 4 Urinary TCS concentrations and stimulation outcomes.

\begin{tabular}{|c|c|c|c|}
\hline & TCS $<$ median $(n=72)$ & TCS $\geq$ median $(n=72)$ & $\boldsymbol{P}$ \\
\hline Mean age (years) & $32.2(4.7)$ & $30.6(3.9)$ & 0.028 \\
\hline Body mass index (BMI) $\left(\mathrm{kg} / \mathrm{m}^{2}\right)$ & $21.7(2.7)$ & $21.6(2.8)$ & 0.772 \\
\hline Menstrual cycle duration (days) & $33.7(15.2)$ & $37.0(21.7)$ & 0.281 \\
\hline Total antral follicle count & $12.0(6.4)$ & $15.4(8.6)$ & 0.008 \\
\hline Baseline FSH (IU/mL) & $7.6(3.3)$ & $6.9(1.7)$ & 0.098 \\
\hline Baseline LH (IU/mL) & $5.5(3.3)$ & $5.9(4.3)$ & 0.483 \\
\hline Baseline LH/FSH & $0.8(0.4)$ & $0.9(0.7)$ & 0.163 \\
\hline Baseline E2 (pg/mL) & $43.2(32.4)$ & $46.3(20.4)$ & 0.489 \\
\hline Baseline testosterone $(\mathrm{ng} / \mathrm{mL})$ & $1.0(0.9)$ & $0.4(0.3)$ & 0.349 \\
\hline Total dose of gonadotrophins (IU) & $2511.3(1211.1)$ & $2232.8(1070.9)$ & 0.148 \\
\hline E2 on HCG $(\mathrm{pg} / \mathrm{mL})$ & $3237.9(1871.0)$ & $4129.6(2554.4)$ & 0.046 \\
\hline Number of oocytes retrieved & $12.3(7.5)$ & $14.4(6.6)$ & 0.031 \\
\hline Number of MII oocytes & $9.7(7.7)$ & $11.4(6.6)$ & 0.037 \\
\hline Number of fertilized eggs & $9.8(7.1)$ & $10.5(5.2)$ & 0.487 \\
\hline Number of $2 \mathrm{PN}$ eggs & $7.6(5.4)$ & $8.3(4.3)$ & 0.384 \\
\hline Number of top quality embryos & $3.7(3.1)$ & $3.4(2.7)$ & 0.514 \\
\hline Number of transferred embryos & $2.0(0.4)$ & $2.0(0.1)$ & 0.745 \\
\hline Oocyte maturation rate $(\%)$ & $78.8 \%$ & $79.3 \%$ & 0.784 \\
\hline Fertilization rate $(\%)$ & $79.7 \%$ & $73.0 \%$ & 0.001 \\
\hline Normal fertilization rate (\%) & $61.9 \%$ & $57.8 \%$ & 0.064 \\
\hline Top quality embryo formation rate (\%) & $48.9 \%$ & $40.9 \%$ & 0.006 \\
\hline Clinical pregnancy rate $(\%)$ per cycle & $52.8 \%(n=62)$ & $47.2 \%(n=61)$ & 0.401 \\
\hline Implantation rate $(\%)$ per embryo & $50.0 \%(n=62)$ & $27.6 \%(n=61)$ & 0.000 \\
\hline
\end{tabular}




\section{Discussion}

It has been reported that TCS could be detected in approximately $75 \%$ of urine samples of US population (Calafat et al. 2008) and the geometric mean (GM) concentration was $12.7 \mu \mathrm{g} / \mathrm{g} \mathrm{Cr}$ (equivalent to $4.96 \mu \mathrm{mol} /$ mol Cr). In another survey (Li et al. 2013) of 287 participants aged between 3 and 24 years in Guangzhou (China), the detection rate of urinary TCS was 93\% and the $\mathrm{GM}$ concentration was $3.77 \mu \mathrm{g} / \mathrm{g} \mathrm{Cr}$ (equivalent to $1.47 \mu \mathrm{mol} / \mathrm{mol} \mathrm{Cr}$ ). In our study, the detection rate of TCS was $60.4 \%(87 / 144)$ and the GM concentration was $0.041 \mu \mathrm{mol} / \mathrm{mol} \mathrm{Cr}$, which is lower than the level previously reported. The subjects in our study were subfertile patients seeking assisted reproductive treatments. In view of their subfertility, all patients had been educated and informed by the physicians and nurses from another lower-level hospital before transferring to our institution. They understood the harm of pollutants and made efforts to reduce exposure to possible pollutants, which might explain why the urinary concentration is lower in this specific population.

In our study, aquaculture food intake and cosmetic use were two variables associated with higher TCS exposure. It has been established that TCS is present in personal care products and household items, such as shampoo, toothpaste, dishwashing detergent, antimicrobial agent, cosmetics and so on (Dhillon et al. 2015). Such products are externally applied to the human body and pose no immediate threat to one's health as there is no ingestion of TCS. However, the release of such products into domestic wastewater may end up in the accumulation of TCS in aquatic animals and plants, and long-term consumption of these products might be one of the main sources of chronic human exposure to TCS (Dann \& Hontela 2010, Escarrone et al. 2016, Nadia et al. 2016). Indeed, $95.1 \%(137 / 144)$ of the participants in our study were aquaculture food consumers, and a positive dose-response association could be observed between the amount of aquaculture food intake and urinary TCS concentrations $(P<0.001)$.

Due to its molecular structure, which closely resembles anthropogenic estrogens, TCS is highly suspected to be an endocrine-disrupting chemical (EDC) (Dhillon et al. 2015, Wang \& Tian 2015, Yuan et al. 2015). However, the correlation between the concentration of TCS and female infertility remains unclear due to lack of studies available. In our study, although no significant difference was observed in oocyte maturation rate, top embryo formation rate and implantation rate was significantly influenced by higher TCS exposure, and a decreased trend of fertilization rate could be observed. Our results are consistent with some previous reports. In Hwang's study on sea urchin, when both eggs and sperms were exposed to TCS, fertilization rate was affected at $0.5 \mu \mathrm{M}$ of TCS and when the concentration was higher than $1.25 \mu \mathrm{M}$, fertilization could not occur spontaneously.
In the same study, early embryo development was significantly inhibited when exposed to $1.0 \mu \mathrm{M}$ TCS (Hwang et al. 2014). Another in vivo study with mice indicated a decrease of implantation sites in the high dose group (18 and $27 \mathrm{mg} /$ animal/day), additionally a combined bisphenol A (BPA) and TCS dosing at individually ineffective doses on gestational day 1-3 significantly inhibited embryo implantation (Crawford \& Decatanzaro 2012). In the present study, we also observed a significant increase of AFC in patients who had higher urinary TCS concentrations after age, BMI and smoking status adjustment. It has been reported that EDCs, such as BPA, may play a role in the onset of polycystic ovary syndrome, which could significantly contribute to female infertility due to anovulation (Barrett \& Sobolewski 2014). As the molecular structure of TCS is quite similar to that of BPA, we hypothesized that TCS may also exert similar detrimental effects on human reproduction. However, in our study, no statistical difference was observed in the menstrual cycle duration, baseline testosterone or $\mathrm{LH} / \mathrm{FSH}$ ratio.

The potential limitations of our study are the small sample size and the two spot sampling method. TCS has a short half-life, which could be totally excreted from the human body in less than $24 \mathrm{~h}$ after exposure (Sandborgh-Englund et al. 2006). In our study, although two spot urine samples were collected, misclassification of TCS exposure is still possible due to patients' highly variable diet and lifestyle habits. Unfortunately, we were unable to assess the TCS exposure profile of the male partners. Therefore, we could only speculate but are unable to determine whether the decreased fertilization rate is due to the toxic effects of TCS on oocyte, sperm or both gametes. To the best of our knowledge, this is one of the first prospective studies designed to evaluate the association between TCS exposure and early IVF outcomes in women with fertility problems.

In conclusion, we observed that there were adverse associations between urinary TCS concentration and early reproductive outcomes of patients undergoing IVF-ET. Women with higher urinary TCS concentration had significantly lower top embryo formation and implantation rates, with a significant increase in baseline AFC after adjustment for age, BMI and smoking status. Further research on plausible mechanisms of TCS reproductive endocrine-disrupting effects is warranted. The estimated TCS exposure for humans $(0.13 \mathrm{mg} / \mathrm{kg} /$ day (Rotroff et al. 2010)) is significantly lower than that used in existing animal studies $(300 \mathrm{mg} / \mathrm{kg} /$ day $)$. Therefore, studies using significantly lower doses of TCS are required to explore the exact effects of TCS on humans such that appropriate public health messages with accurate facts can be presented, and major lifestyle modifications could be made in subfertile patients depending on the future findings of such investigations. 


\section{Declaration of interest}

The authors declare that there is no conflict of interest that could be perceived as prejudicing the impartiality of the research reported.

\section{Funding}

This work was supported by National Plan for Science and Technology Research (2013BAI12B00).

\section{Acknowledgements}

We gratefully acknowledge Pei Li and Zhiqiang Yu for their technical assistance with TCS measurements. We also acknowledge the National Plan for Science and Technology Research (2013BAI12B00) for supporting our work. Last but not the least, we highly appreciate all the participants in our study.

\section{References}

Ahn KC, Zhao B, Chen J, Cherednichenko G, Sanmarti E, Denison MS, Lasley B, Pessah IN, Kultz D, Chang DP et al. 2008 In vitro biologic activities of the antimicrobials triclocarban, its analogs, and triclosan in bioassay screens: receptor-based bioassay screens. Environmental Health Perspectives 116 1203-1210. (doi:10.1289/ehp.11200)

Allmyr M, Adolfsson-Erici M, McLachlan MS \& Sandborgh-Englund G 2006a Triclosan in plasma and milk from Swedish nursing mothers and their exposure via personal care products. Science of the Total Environment 372 87-93. (doi:10.1016/j.scitotenv.2006.08.007)

Allmyr M, McLachlan MS, Sandborgh-Englund G \& Adolfsson-Erici M $2006 b$ Determination of triclosan as its pentafluorobenzoyl ester in human plasma and milk using electron capture negative ionization mass spectrometry. Analytical Chemistry 78 6542-6546. (doi:10.1021/ ac060666x)

Escarrone AL, Caldas SS, Primel EG, Martins SE \& Nery LE 2016 Uptake, tissue distribution and depuration of triclosan in the guppy Poecilia vivipara acclimated to freshwater. Science of the Total Environment 560-561 218-224. (doi:10.1016/j.scitotenv.2016.04.039)

Dann AB \& Hontela A 2010 Triclosan: environmental exposure, toxicity and mechanisms of action. Journal of Applied Toxicology 31 285-311. (doi:10.1002/jat.1660)

Axelstad M, Boberg J, Vinggaard AM, Christiansen S \& Hass U 2013 Triclosan exposure reduces thyroxine levels in pregnant and lactating rat dams and in directly exposed offspring. Food and Chemical Toxicology 59 534-540. (doi:10.1016/j.fct.2013.06.050)

Barrett ES \& Sobolewski M 2014 Polycystic ovary syndrome: do endocrinedisrupting chemicals play a role? Seminars in Reproductive Medicine 32 166-176. (doi:10.1055/s-0034-1371088)

Buttke DE, Schier JG, Bronstein AC \& Chang A 2012 Characterization of animal exposure calls captured by the national poison data system, 2000-2010. Journal of Clinical Toxicology 2 117. (doi:10.4172/21610495.1000117)

Calafat AM, Ye X, Wong LY, Reidy JA \& Needham LL 2008 Urinary concentrations of triclosan in the U.S. population: 2003-2004. Environmental Health Perspectives 116 303-307. (doi:10.1289/ ehp.10768)

Chen M, Tang R, Fu G, Xu B, Zhu P, Qiao S, Chen X, Xu B, Qin Y, Lu C et al. 2013 Association of exposure to phenols and idiopathic male infertility. Journal of Hazardous Materials 250-251 115-121. (doi:10.1016/j. jhazmat.2013.01.061)

Christen V, Crettaz P, Oberli-Schrammli A \& Fent K 2010 Some flame retardants and the antimicrobials triclosan and triclocarban enhance the androgenic activity in vitro. Chemosphere 81 1245-1252. (doi:10.1016/j.chemosphere.2010.09.031)
Crawford BR \& Decatanzaro D 2012 Disruption of blastocyst implantation by triclosan in mice: impacts of repeated and acute doses and combination with bisphenol-A. Reproductive Toxicology 34 607-613. (doi:10.1016/j.reprotox.2012.09.008)

Dhillon GS, Kaur S, Pulicharla R, Brar SK, Cledon M, Verma M \& Surampalli RY 2015 Triclosan: current status, occurrence, environmental risks and bioaccumulation potential. International Journal of Environmental Research and Public Health 12 5657-5684. (doi:10.3390/ ijerph120505657)

Forgacs AL, Ding Q, Jaremba RG, Huhtaniemi IT, Rahman NA \& Zacharewski TR 2012 BLTK1 murine Leydig cells: a novel steroidogenic model for evaluating the effects of reproductive and developmental toxicants. Toxicological Sciences 127 391-402. (doi:10.1093/toxsci/ kfs121)

Gee RH, Charles A, Taylor N \& Darbre PD 2008 Oestrogenic and androgenic activity of triclosan in breast cancer cells. Journal of Applied Toxicology 28 78-91. (doi:10.1002/jat.1316)

Huang H, Du G, Zhang W, Hu J, Wu D, Song L, Xia Y \& Wang X 2014 The in vitro estrogenic activities of triclosan and triclocarban. Journal of Applied Toxicology 34 1060-1067. (doi:10.1002/jat.3012)

Hwang J, Suh SS, Chang M, Yun Park S, Ryu TK, Lee S \& Lee TK 2014 Effects of triclosan on reproductive parameters and embryonic development of sea urchin, Strongylocentrotus nudus. Ecotoxicology and Environmental Safety 100 148-152. (doi:10.1016/j.ecoenv.2013.10.029)

Johnson PI, Koustas E, Vesterinen HM, Sutton P, Atchley DS, Kim AN, Campbell M, Donald JM, Sen S, Bero L et al. 2016 Application of the Navigation Guide systematic review methodology to the evidence for developmental and reproductive toxicity of triclosan. Environment International 92-93 716-728. (doi:10.1016/j.envint.2016.03.009)

Kumar V, Balomajumder C \& Roy P 2008 Disruption of LH-induced testosterone biosynthesis in testicular Leydig cells by triclosan: probable mechanism of action. Toxicology 250 124-131. (doi:10.1016/j. tox.2008.06.012)

Li X, Ying GG, Zhao JL, Chen ZF, Lai HJ \& Su HC 2013 4-Nonylphenol, bisphenol-A and triclosan levels in human urine of children and students in China, and the effects of drinking these bottled materials on the levels. Environment International 52 81-86. (doi:10.1016/j.envint.2011.03.026)

Moss T, Howes D \& Williams FM 2000 Percutaneous penetration and dermal metabolism of triclosan (2,4,4'-trichloro-2'-hydroxydiphenyl ether). Food and Chemical Toxicology 38 361-370. (doi:10.1016/S02786915(99)00164-7)

Mustafa M, Wondimu B, Hultenby K, Yucel-Lindberg T \& Modeer T 2003 Uptake, distribution and release of 14C-triclosan in human gingival fibroblasts. Journal of Pharmaceutical Sciences 92 1648-1653. (doi:10.1002/jps.10429)

Nadia Carmosini, Sarah Grandstrand \& Tisha C King-Heiden 2016 Developmental toxicity of triclosan in the presence of dissolved organic carbon: moving beyond standard acute toxicity assays to understand ecotoxicological risk. Zebrafish 13 424-431. (doi:10.1089/ zeb.2015.1220)

Provencher G, Berube R, Dumas P, Bienvenu JF, Gaudreau E, Belanger P \& Ayotte P 2014 Determination of bisphenol A, triclosan and their metabolites in human urine using isotope-dilution liquid chromatography-tandem mass spectrometry. Journal of Chromatography A 1348 97-104. (doi:10.1016/j.chroma.2014.04.072)

Queckenberg C, Meins J, Wachall B, Doroshyenko O, TomalikScharte D, Bastian B, Abdel-Tawab M \& Fuhr U 2010 Absorption, pharmacokinetics, and safety of triclosan after dermal administration. Antimicrobial Agents and Chemotherapy 54 570-572. (doi:10.1128/ AAC.00615-09)

Rotroff DM, Wetmore BA, Dix DJ, Ferguson SS, Clewell HJ, Houck KA, Lecluyse EL, Andersen ME, Judson RS, Smith CM et al. 2010 Incorporating human dosimetry and exposure into high-throughput in vitro toxicity screening. Toxicological Sciences 117 348-358. (doi:10.1093/toxsci/ kfq220)

Sandborgh-Englund G, Adolfsson-Erici M, Odham G \& Ekstrand J 2006 Pharmacokinetics of triclosan following oral ingestion in humans. Journal of Toxicology and Environmental Health, Part A 69 1861-1873. (doi:10.1080/15287390600631706)

Stoker TE, Gibson EK \& Zorrilla LM 2010 Triclosan exposure modulates estrogen-dependent responses in the female wistar rat. Toxicological Sciences 117 45-53. (doi:10.1093/toxsci/kfq180) 
Wang CF \& Tian Y 2015 Reproductive endocrine-disrupting effects of triclosan: population exposure, present evidence and potential mechanisms. Environmental Pollution 206 195-201. (doi:10.1016/j. envpol.2015.07.001)

Yuan M, Bai MZ, Huang XF, Zhang Y, Liu J, Hu MH, Zheng WQ \& Jin F 2015 Preimplantation exposure to bisphenol a and triclosan may lead to implantation failure in humans. BioMed Research International 2015 184845. (doi:10.1155/2015/184845)

Zorrilla LM, Gibson EK, Jeffay SC, Crofton KM, Setzer WR, Cooper RL \& Stoker TE 2009 The effects of triclosan on puberty and thyroid hormones in male Wistar rats. Toxicological Sciences 107 56-64. (doi:10.1093/ toxsci/kfn225)

Received 13 September 2016

First decision 10 October 2016

Revised manuscript received 11 December 2016

Accepted 19 December 2016 